\title{
Hauptwürm, Spätwürm, Frühwürm und die Frage der älteren Würmschotter
}

Ein Beitrag zur Gliederung der Würmeiszeit aus dem bayrischen Salzachvorlandgletscher

\author{
Von Edrth Ebers, München
}

Mit 3 Abb.

Zusammenfassung. Es läßt sich, wenn man das Grundsätzliche herausschält, am Salzachvorlandgletscher eine Abfolge im Geschehen der Würmzeit herauslesen, die wohl allgemeinere Bedeutung hat. Der Innvorlandgletscher, der nur wie ein größerer Bruder des Salzachvorlandgletschers war, bietet zahlreiche Entsprechungen und Vergleichsmomente. Das überfahrene Frühw ürm, auf das ein Rückzug bis an den Alpenrand folgte, die La ufenschwankungoderdas A urignacInterstadial, das große Hauptwürmmit seinendrei Endmoränensystemen und dazugehörigen Erscheinungen und der besonders deutlich $\mathrm{klima-}$ $\mathrm{t}$ is ch bestimmte Einschnit des beginnenden $S \mathrm{pätw}$ ü $\mathrm{rm}$ mit den peripher gesammelten Schmelzwässern, den ersten Eissta useen und späteren $\mathrm{Schmelz-}$ wasserseen sind wahrscheinlich in allen nordalpinen Vorlandgletschern wieder zu erkennen ${ }^{1}$ ).

Abstract. During the last Alpine glaciation (Würm) there have been a number of Piedmont glaciers protruding from the main Alpine valleys and extending like big fans over the northern foreland in Bavaria. Two of this Piedmont glaciers can be compared and coordinated with each other: the Inn-Piedmont glacier and its eastern neighbour, the Salzach Piedmont glacier.

Early Würm glaciation. After a first Würm stage with moraines, deltas and gravel deposits, regionally covered by a younger boulder clay, the ice edge retreated to the foot of the Alps ("Laufenschwankung", Aurignac interval).

$\mathrm{Ma}$ in Würm glaciation. After this interval the glaciers advanced a second time (Upper Laufen gravel deposits with Elephas primigenius in great number and ice wedges), covered all deposits of the former early Würm stage and reached their Würm maximal stand (3 systems of moraines and outwash fans).

L a te Würm time. Later on, when the climate improved and the glaciers melted on a big scale marginal drainage and a number of big lakes developed. In the beginning of this meltwater period the lakes were dammed by the retreating ice edge at their proximal side.

\section{Ha u ptw ü $r m$}

Ein Rückzug bis in das Alpengebirge - also ein Interstadium - kommt im Bereiche des Salzachvorlandgletschers während der drei Hauptphasen der Würmeiszeit nicht in

1) Die örtlichen Bezeichnungen für den Salzachgletscherlobus sind zwecks leichterer Nachprüfung von besonders charakteristischen Punkten gewählt. Zur Erleichterung des Verständnisses sind die Troll'schen und KNaUer'schen Bezeichnungen übernommen. Als "Phasen“ werden Randlagen, als "Stadien“ Neuvorstöße bezeichnet. Der Internationale Quartärkongreß in Italien 1953 zeigte wieder die mißverständliche, in der Literatur schon verbreitete Verwendung einer Zifferngliederung von Würm, ohne daß die entsprechenden Parallelisierungen in allen Fällen sicher möglich wären. Auf eine solche Bezeichnungsart wird hier deshalb verzichtet.

Das Schriftenverzeichnis führt Schriften von J. BüDel, C. Rathjens, J. SchaEfER u. a. an, die in den letzten Jahren Beiträge zu der Frage eines Würm-Interstadials geleistet haben. 
Frage. Diese drei Hauptphasen sind die der räumlich größten Ausdehnung des Würmeises. Sie korrespondieren mit den Phasen des Inngletschers nach C. TROLL:

$\begin{array}{lll}\text { Salzachgletscher } & \text { Inngletscher } & \text { Z ugehörige Terr.-Stufe n } \\ \text { Unterweißenkirchen } & \text { Aying } & \text { Wolfersberg-Neustadler } \\ \text { Nunreut } & \text { Kirchseeon } & \text { Aicher, Kirchreiter, Trauntal } \\ \text { Radegund } & \text { Ebersberg } & \text { Trauntaler, Rauschinger }\end{array}$

Diese drei Stillstandslagen werden durch räumlich und zeitlich relativ wenig voneinander getrennte Randbildungen von Satz- oder Stillstandsmoränen belegt. Die Terrassenmorphologie bei Tyrlaching-Schnitzing zeigt mit voller Deutlichkeit, daß die Terrassen ohne größeren Unterbruch nacheinander entstanden. Drei nur wenig von*einander verschiedene Terrassenstufen sind hier in einem heute trockenen Tälchen, dem „Schnitzinger Schmelzwassertal“, untereinander eingeschachtelt. Die normale Beziehung $\mathrm{zu}$ den entsprechenden Moränenwällen ist in diesem Raume besonders klar zu erkennen.

Die vorgeschobene $U$ nterweißenkirchner Außenphase von Hauptwürm ist nicht nur durch ihre Terrassenreste, sondern auch durch Anschnitte an der Proximalseite ihrer geringmächtigen Moränen, welche durch die Schmelzwässer der proximalwärts folgenden Nunreuter Hauptphase hervorgebracht wurden, deutlich abzutrennen. Die im Gegensatz hierzu bedeutenden $\mathrm{Nun} r$ e u te r und nach einwärts anschließenden $\mathrm{R}$ a degunder $\mathrm{Ha}$ u t moränenwälle wachsen an manchen Stellen zu einer mächtigen Einheit zusammen (z. B. bei Bergham-Kofeln), lassen sich aber auch dann durch die Ansatzstelle der Trauntalstufe bei Meggenthal und das zugehörige Trompetentälchen voneinander trennen. Während dieser drei Moränenaufschüttungen, welche die Hauptwürmvergletscherung repräsentieren, ist die Entwässerung zentrifugal nach außen gerichtet, wenn sich auch während der dritten, der Radegunder Phase, schon eine gewisse Tendenz zum Übergang in die periphere Richtung einstellt.

\section{II. $S \mathrm{p} \ddot{\mathrm{t}} \mathrm{t}$ ü $\mathrm{r} \mathrm{m}$}

Proximalwärts dieser drei Maximalphasen folgt das Ollerdinger E is r a n dt a 1, ein echtes Urstromtal, jedoch nicht durchgängig mit ausgeglichenem Längsprofil und Terrassen ausgestaltet, wie das weiter distalwärts liegende Schnitzinger Schmelzwassertal, in welchem die Schmelzwässer der drei Hauptphasen die peripher zum Würmmoränenrund angeordnete Hochterrasse des westlichen Salzachgletscherflïgels durchbrachen, um zur $\mathrm{Alz}$ zu gelangen. Das Ollerdinger Eisrandtal entspricht dem Leizach-Gars-Talzug im Inngletscher; aber keine Mangfall konnte hier einbrechen (siehe J. KNaUER 1937, S. 28). Es setzt sich vielmehr aus einzelnen, teils durch Erosion, teils durch lokale Akkumulation ausgestalteten Abschnitten zusammen, deren formende schotterarme Schmelzwässer von Gletschertoren ausgingen, die an dem proximalwärts liegenden, vierten Moränenwall wurzelten. Terrassierung ist in diesem Tälchen nicht vorhanden. Erst bei Olle rding-Lanzing verebnet sich der Talboden mehr und mehr und erweitert sich zu der Deltaebene des Tit tmoninger Eissta usees. (Abb. 1).

"Große Seenzeit “. Dieser Tittmoninger See dürfte der Ebinger Stufe C. 'Troll's zeitlich entsprechen. Er spiegelte in einer Höhe von $465-475 \mathrm{~m}$ und ist der älteste und höchste Stausee des Gebietes. Der Eisrand muß noch nahe gelegen haben, wie Grundmoränenschollen in den mächtigen Deltakiesen von Tittmoning erkennen lassen. Riesige kantige Phyllitblöcke aus der Schieferhülle der Hohen Tauern drifteten auf Eisschollen in die Seeablagerungen hinein und liegen jetzt als Überrest abgebauter Schotterpartien häufig in den Kiesgruben N Tittmoning auf der Deltaebene.

Das Ollerdinger Eisrandtal und der Tittmoninger Eisstausee lagen auf der Distalseite jener vierten frischen Randlage, die durch junge, typisch geformte Endmoränen-

7 Eiszeit und Gegenwart 
kuppen, z. B. beim Bahnhof und dem Kirchlein von Lanzing, gekennzeichnet ist. Es sind die Moränen der Lanzinger (O lkofner) Phase. Der Stausee erfüllte einen wahrscheinlich nur schmalen Zipfel am Nordende des Tittmoninger Zweigbeckens. Auf der bayrischen Seite lag das Nordende des Tittmoninger Eisstausees beim Weiler Enzelsberg; hier stauten ihn die Moränen der Würmhauptphasen. Im Süden staute ihn die nicht allzu weit entfernte Eismauer.

Die Moränen der Lanzinger Phase liegen auf einem älteren Beckenrand. Die Lanzinger Phase ist dadurch gekennzeichnet, daß sie keine zentrifugal entweichenden Schmelzwässer mehr besaß. J. BüDEL (1950) hebt hervor, daß die entsprechende Ölkofner Phase in anderen voralpinen Vereisungsgebieten, wie Iller- und Rheingletscher, auch noch zentrifugal nach außen ziehende Schotterstränge besitzt. Im Salzachgebiet fehlen ihr Sander, Trompetentälchen usw. Mit dieser Phase beginnt die Große Seenzeit des nördlichen Alpenvorlandes. Die Gewässer ziehen peripher am Eisrand entlang - dabei zeitweise zu Seen aufgestaut - zu einer Hauptsammelader hin und beginnen den heutigen $\mathrm{Salzach}-\mathrm{Can}$ y on zwischen Tittmoning und Burghausen anzulegen. Diese Hauptsammelader war zugleich die älteste würmspätglaziale Salzach und der Abfluß des ältesten und höchsten Stausees. Zur HauptwürmGlazialzeit - also während der drei Phasen von Hauptwürm - war die Ursalzach, wie schon Penck-Brückner annahmen - im Gebiete des heutigen Weilhart-Forstes in

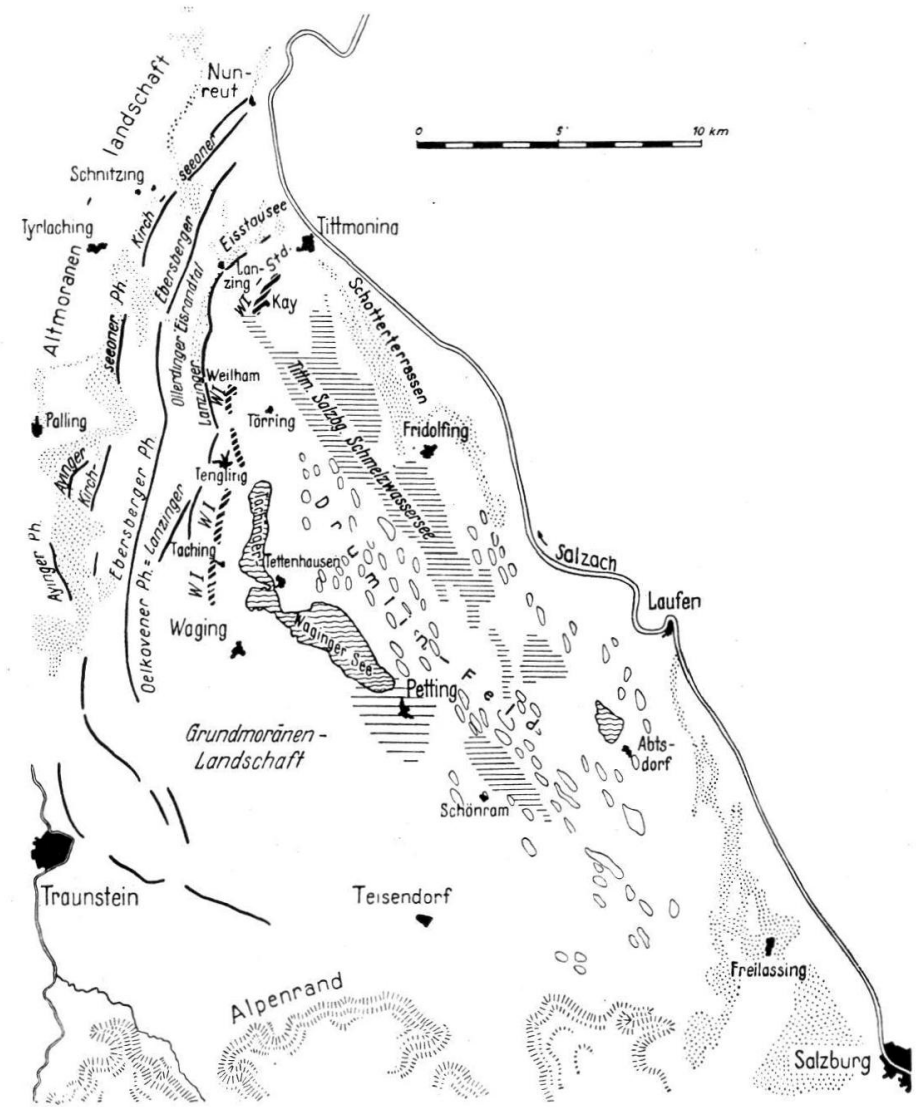

Abb. 1. Endmoränen-Lagen auf dem Westflügel des Salzach-Vorlandgletschers.

Taching liegt am See 
Oberösterreich abgeflossen; der westliche Salzachgletscherflügel entwässerte währenddessen durch das bereits erwähnte Schnitzinger Schmelzwassertal.

Während der Lanzinger Phase und der Großen Seenzeit standen zweifellos bedeutende Schmelzwassermengen zur Verfügung. Eine einschneidende Klimaänderung gegenüber dem hocharktischen und als arider anzusehenden Hauptwürm, das kalt-kontinentalen Klimacharakter trug, ist nun offenbar geworden.

Die großen Schmelzwasserseen der Wörther und Pürtener $\mathrm{Stufe}$. C. Troll faßt in seiner Darstellung des Inngletschers die spätglazialen Seen zu. einem einzigen großen „Rosenheimer See“ zusammen. Er spricht von höheren Seeständen nur in örtlich gestauten Zweigbecken. Im Salzachgebiet folgten mehrere weiter absinkende Stammbeckenseen aufeinander. Das Tittmoninger Zweigbecken stellt nur eine Verlängerung des Salzburger Stammbeckens dar. Der Seespiegel, der durch das Tittmoninger Delta angezeigt wird, lag auf mindestens $465 \mathrm{~m}$ ü. M. Eis erfüllte noch das Stammbecken.

Salzburg-Tittmoninger Seen I und II. Nun aber reichen Seetone im Stammbecken von Tittmoning-Salzburg bis nach Salzburg zurïck. Das oberste Seetonniveau im Salzburg-Tittmoninger Stammbecken beträgt $425-440 \mathrm{~m}$. Eisstauung schon in der Nähe des Alpenrandes - mag auch hier noch mitgewirkt haben. Bei Tittmoning bleibt der Seeton, der heute - infolge der Salzachkorrektion - überhaupr nicht mehr zu sehen ist, aber von E. BRüCKNER „N Tittmoning“ noch gesehen wurde, tief unter dem oben am Beckenrand hängenden Delta zurück. Verzahnungen der Deltasedimente mit Seetonen sind nicht bekannt, und die Deltaschüttung reicht nicht bis ins Becken hinein. Bei den Seetonen von Tittmoning haben wir es schon mit einem zweiten Seespiegelniveau (Schmelzwassersee I) zu tun, das sich C. Troll's Wörther Stufe zuordnet (bis $440 \mathrm{~m}$ ) oder einem dritten (Schmelzwassersee II), welches um Fridolfing-Ostermiething ausgedehnte Seetonebenen zurückließ. Dies Seetonniveau liegt auf 410-420 m. Man kann - mit anderen Autoren - annehmen, daß die Seetone die spätglazialen Seen fast ganz auffüllten. Diese beiden Seetonniveaus reichen auch in mehrere der österreichischen Zweigbecken hinein (Ibmer Moos, Oichtental; E. Ebers 1932). Bedeutende Mächtigkeit der Tone wurde bei Bohrungen auf tertiäre Kohle in den letzten Jahren immer wieder festgestellt.

Durchbruch von Laufen. Im spätglazial entstandenen Durchbruch von Laufen liegt die Terrasse von Osing in $415 \mathrm{~m}$ ï. M. Auf der österreichischen Seite sind noch etwas höhere Terrassenreste vorhanden. In Zeiten einer Spiegelhöhe von 410 bis $420 \mathrm{~m}$ war also der Salzburg-Tittmoninger-Schmelzwassersee schon in zwei Seen zerfallen, von welchen einer das südliche Salzburger und einer das nördliche Tittmoninger Becken erfüllte. Eine Flußstrecke, welche die Laufenschotterbarre durchbrach, verband sie. In diesem Stadium muß der Eisrand schon am Alpenrand oder im inneralpinen Salzachtal gelegen und der See bis mindestens Salzburg gereicht haben. Der Autobahnbau erschloß bei Liefering (Salzburg) gebänderte Seetone. Die Lage dieser Gletscherstirn würde der PENcK'schen „Bühlzeit" entsprechen. Es ist die Zeit der Pü r te ne r Stufe, und der Salzachgletscher muß nun den Anblick eines inneralpinen „Fjordgletschers" geboten haben.

Ungeheure Massen von Gletschertrübe, auch Seesande, wurden während dieser beiden Seestände ins Alpenvorland vertragen. Die Seetone (Kalkmergel) werden außerordentlich mächtig, sie sind in den basalen Partien, die meist auf Grundmoräne liegen, häufig schön gebändert; die Jahreswarven können nach oben hin sehr dick werden. Rippelmarken sind zu sehen. Nicht nur bei Fridolfing-Götzing auf dem bayrischen Sektor, sondern auch im oberösterreichischen Teil des Salzachgletschers sind Lagen mit Konkretionen nach Wurzeln von Wasserpflanzen zu finden. Die in früheren Jahren sehr schön zu beobachtenden Würgeboden-ähnlichen Strukturen in den hangenden La- 
gen am ehemaligen Seeufer dürften eher auf subaquatische Rutschungen zurückzuführen sein. Eine Probenfolge der Seetonsedimente von Fridolfing-Götzing liegt beim Amt für Bodenforschung in Krefeld und soll dort pollenanalytisch untersucht werden.

Beginn der Spätglazia l-Zeit. Die peripheren Täler und die Ablagerungen der ersten großen Stauseen in den Hauptbecken der verschiedenen voralpinen Gletschergebiete müssen oft leichter zu parallelisieren sein als dic einzelnen Moränenzüge, und mit ihnen möchte man -, nach dem Abschluß von Hauptwürm-, Spätwürm beginnen lassen. Diese Festsetzung würde mit derjenigen von P. Woldstedt u. a. übereinstimmen, welche den Beginn des Spätglazials an den Schluß des Pommerschen Stadiums stellt. Zu dieser Zeit war auch in Norddeutschland die Entwässerung neue Wege gegangen, und der Westteil des europäischen Inlandeises entwässerte, mindestens zum Teil, subglaziär zum Kattegat hin, oder, wo dies nicht der Fall war, ersetzten, ähnlich wie für das Alpenvorland oben dargelegt, Schmelzwassertäler die Sander. Wo aber noch die alte, zentrifugale Entwässerung bestand, wie besonders im Ostteil des Inlandeises, waren auch Sander entwickelt. Übrigens vermutet K. GRIPP (1940), daß die zentripetale Umkehr der Hydrographie im Alpenvorland subglaziär schon während der Ölkofner Phase einsetzte.

Dieser Vorschlag, die Spätglazialzeit im Alpenvorland mit der „Großen Seenzeit" und dem Rückzug von der Ölkofner Phase beginnen zu lassen, deckt sich nicht mit demjenigen von J. Schaefer (1953), den Einschnitt zwischen Hochglazial und Spätglazial schon in die Zeit des Rückzuges von der äußersten Randlage von Hauptwürm zu legen. Konsequenterweise müßte es sich im Salzachgebiet dabei um die kleine, aber am weitesten vorgeschobene Randlage der Unterweißenkirchner Phase handeln. Ihr folgten aber erst die großen Hauptphasen von Würm, denen von allen Seiten jetzt ein kalt-kontinentales, hochglaziales Klima zugeschrieben wird. Die Anzeichen eines entscheidenden Klimaumschwunges zeigen sich erst beim Beginn der Entwicklung großer Stauseen, welche gewaltige Schmelzwassermengen und einen schnellen Eisrückgang erkennen lassen.

End-W ürm. Unter diesem tiefsten Spiegelniveau des Salzburg-Tittmoninger Sees hat dann, wie C. Troll es auch von Rosenheim und vom Inngletscher entsprechend beschreibt, die Salzach noch zwei ausgedehnte Schotterstufen, die Gwe nger und die Niederndorfer $S t u f e$ ausgebildet. Die erstere ist in die Seeablagerungen eingeschnitten. Eiszeitliche Klimabedingungen haben zu dieser Zeit im Alpenvorland nicht mehr geherrscht. Wiederbewaldung bis an den Alpenrand hin muß möglich gewesen sein ${ }^{2}$ ). E. SEEFELDNER (1954) hat Anhaltspunkte dafür, daß die Salzburger Schotterebene, welche diesen letzten Stufen entsprechen muß, aus der Zeit des Schlernstadiums stammt (Alter 11000 Jahre).

III. Fr ühw ürm

Wenn die Kuppenreihen der Lanzinger (Olkofner) Phase auch eine bedeutende, frische Moränenrandlage darstellen, so ist damit doch noch nicht gesagt, was unter ihnen und weiter proximalwärts liegt. Bei Schlaffen (Ollerding) kommt darunter diskordant frische Grundmoräne zutage. Vermutlich ordnet sie sich in eine Grundmoränendecke von nur wenigen Metern Mächtigkeit ein, wie sie weiterhin ein Kennzeichen der Landoberflächen ist, die proximalwärts das Beckengebiet des Tachingersees begrenzen. Sie bedeckt die plumpen breiten Kuppen, die hier aufragen. Sie bildet auch die in Stromlinienkörperform angeschmiegte Decke der Drumlins, soweit sie Schotterkerne besitzen, oder schwillt zum Aufbau des ganzen Drumlinkörpers an. Diese

$\left.{ }^{2}\right)$ Die Frage des Spätwürm-Interstadials (Alleröd) konnte hier nicht mehr miteinbezogen werden. Ebenso ist es nicht möglich, die deutsch-österreichische Lößstratigraphie, die noch im Stadium der Polemik steckt, miteinzubeziehen. 
diskordant vom Unterlagernden abgetrennte Grundmoränendecke ist zu sehen in den Kiesgruben von Oberstefling, Kay, Tettenhausen, S Schönram und N Taching. Die zugehörigen Aufschlüsse sind teilweise bereits im Jahre 1935 von J. KNAUER (nach Angaben der Verfasserin) in seine Zusammenfassung der Ablagerungen der „älteren Würmeiszeit" (Vorrückungsphase) miteinbezogen worden. In den dazwischen liegenden Jahren tauchten nun auch auf den älteren Würmschottern von Tettenhausen und bei Schönram Reste einer in flache Mulden der Oberfläche eingelagerten diskordanten Grundmoränendecke auf, die vor 1935 noch nicht zu sehen waren. Die Grundmoränendecke liegt bei Taching auf einem älteren, nur gering und örtlich verfestigten, sonst frischen, aber stark gestörten Delta auf $(470-480 \mathrm{~m})$, welches auch oberflächenmorphologisch vollständig in die Grundmoränenlandschaft eingebaut ist. Dies Delta liegt nicht als ein Fremdkörper in der Formenwelt der Grundmoränenlandschaft, wie es die jüngeren, spätglazialen Deltas im Umkreise des Waging-Tachinger Sees tun (Höhe des spätglazialen Waginger Deltas $459 \mathrm{~m}$, heutiger Seespiegel $440 \mathrm{~m}$ ). Diese jüngeren Deltas sind Rückzugsbildungen von Hauptwürm und haben mit den grundmoränenüberdeckten älteren Bildungen nichts zu tun. Sie wurden erst beim endgültigen Eisrückzug in die eisfrei gewordenen Becken eingelagert. Ein dem Tachinger Delta entsprechendes Profil findet sich auch in den Gruben S Schönram im Tale der Sur, wo über grobkörnigen Randterrassenschüttungen, auch mit gestörter Deltaschichtung, zeitweise eine Deckschicht von $1 \mathrm{~m}$ Grundmoräne zu sehen war. Wir befinden uns hier nur noch $7-8 \mathrm{~km}$ vom Alpenrand entfernt.

Es frägt sich nun, was alles sonst noch unter dieser regional verbreiteten Grundmoränendecke, die man dem Hauptwürm zuordnen wird, verborgen liegt. Sicher ist wie eben ausgeführt - daß darunter einmal ältere, würmeiszeitliche Deltas liegen, die leicht und örtlich verfestigt sind, jedoch nicht etwa mit rißeiszeitlichen Deltas verwechselt werden können. Im übrigen sind aber außerdem - so bei Kay und Tettenhausen - als Liegendes der Grundmoränendecke Moränenschotter aufgeschlossen, welche außen oder bankweise zum Teil verfestigt und gelegentlich auch durch ein etwa $10 \mathrm{~cm}$ dickes Konglomeratbändchen (wie dies auch KNAUER 1935 erwähnt) von der hangenden Grundmoräne abgetrennt sind. An den andern genannten Stellen liegt die Grundmoränendecke diskordant über typischen Moränen mit Blöcken und gekritzten Geschieben, so bei Oberstefling NW Waging. Im äußeren Bild handelt es sich dann um breite Kuppen mit ausgeglichenen Oberflächenformen von der Art, wie sie J. KNAUER immer wieder beschrieben hat. Dieses morphologische Bild läßt sich erst im Aufschluß besonders gut verstehen, wo die Grundmoräne in Mulden und flache Wellen der alten Moränenoberfläche hineingedrückt ist. Ist ein solcher Teil der Kiesgrube abgebaut, so kann es unter Umständen Jahre dauern, bis wieder an einer andern Stelle in der Nachbarschaft eine Grundmoränenauflagerung zu sehen ist.

Überfahrenes Würm I-Stadium J. KNAUER's. Die älteren Würmschotter sowie überfahrenen Moränen um den Tachinger See herum sind unzweifelhaft oberhalb des Riß-Würm-Interglazials einzuordnen und gehören einer älteren Würmphase an. Die Schotter können als Vorstoßschotter fluvioglazial, als lakustre Ablagerung (Deltas) beim Rückzug vom überfahrenen W I-Stadium oder auch als fluviatile Schotter des Interstadiums entstanden sein. Am Tachinger See zeigt sich somit (ebenso wie auch bei Unterzeismering am Starnberger See, an den von J. KNAUER angegebenen Stellen, an Schweizer Seen (nach E. BRÜCKNER 1909), daß zur Würmeiszeit schon einmal ein Zyklus von Vorgängen ablief, der mit demjenigen von Hauptwürm und seinen spätglazialen Rückzugserscheinungen grundsätzlich übereinstimmte: Moränen- und Schotterablagerungen und eine Rückzugsphase mit Seenbildung. Dieses ältere Würmstadium ist das überfahrene Würm I-Stadium J. KNAUER's, für welches er in den anderen Vorlandgletschergebieten der Alpen zahlreiche Beweise gefunden hat. Im Salzachgletschergebiet 
bezeichne ich es mit dem Namen "Tenglinger Stadium" und folge dabei auch J. KNaUER. Das Tenglinger Stadium liegt räumlich etwas hinter der Lanzinger (Olkofner) Phase zurück oder wird auch von den Moränen dieser überlagert. Ihm gehören im Salzachgebiet eine Anzahl mächtiger, breiter Kuppen an (Kay, Weilham, Törring, Tengling, Oberstefling), die auf der West- und Nordseite des Tachinger Sees vorbeiziehen. Bei Tettenhausen und Kay sind überfahrene Schotter des Tenglinger Stadiums aufgeschlossen.

Einordnung in Riß III-Eiszeit? J. KNAUER versuchte im Jahre 1938 das überfahrene Würmstadium in eine Riß III-Eiszeit einzuordnen, hat diese Auffassung 1954 aber wieder aufgegeben - zugunsten eines. Würmstadiums. Der ersteren Auffassung könnte ich mich aber auch aus anderen Gründen nicht anschließen. Insbesondere das Fehlen diagenetischer Umwandlungen, nach Art der in Riß-Ablagerungen vorkommenden, in allen Ablagerungen des älteren Würmstadiums und des darauffolgenden Interstadiums ist entscheidend.

Die klimatische Verwitterung des bedeutenden Riß-Würm-Interglazials müßte sie hervorgebracht haben. Das daneben sicherlich viel kürzere und kältere Interstadial konnte solche Erscheinungen nur in kleinerem Maßstab bewirken (siehe J. KNAUER 1937, A. Micheler 1948 und die neuesten Untersuchungen von E. Kraus am „Murnauer Schotter"). Gerade im Salzachgebiet läßt sich in einem bedeutungsvollen Aufschluß in der Niederterrasse bei Palling N Traunstein eine von Niederterrassenschotter und einer mächtigen Verwitterungsschicht bedeckte Terrasse der dritten und letzten Rißphase (nicht Eiszeit!) nachweisen, die in keiner räumlichen Beziehung zu dem überfahrenen Würmstadium steht. Sie läßt die einschneidenden Unterschiede des diagenetischen $\mathrm{Zu}$ standes von Riß- und Würmablagerungen im nördlichen Alpenvorland besonders deutlich erkennen.

Verknetete Lößlehmfetzen an der Basis von Hauptwürm. Die bisher angeführten Beobachtungen im bayrischen Teil des Salzachvorlandgletschers sind nicht die einzigen, welche in jenem Abschnitt des nordalpinen Pleistozäns auf ein älteres überfahrenes Würmstadium hinweisen. Dazu gehören vor allem auch Feststellungen an der Außengrenze der Randlagen von Hauptwürm auf dem östlichen Salzachgletscherflügel in Oberösterreich. Sie wurden teilweise schon von PENCK-BRüCKNER gemacht und knüpfen sich an die Namen der Ortschaften Aschau, Gampern, Feldkirchen, alle in der Nähe von Gundertshausen gelegen. In den basalen Partien der Würmmoränen und Obergangskegel zur Niederterrasse finden sich hier, unmittelbar im Hangenden der Rißmoränen und Hochterrassenschotter, eingeknetete Fetzen von Verwitterungs- und vor allem Lößlehm. Diese Lößablagerung kann nicht dem Hauptwürm zugeordnet werden, da dieses unmittelbar darüber liegt und die Verlehmung des Lösses auf einen zeitlichen Zwischenraum zwischen seiner Ablagerung und Hauptwürm hinweist. Der Löß wird heute allgemein als hochglaziale Bildung angesehen, d. h. als eine Bildung hochglazialen Klimas bei Anwesenheit einer arktischen Steppenvegetation. Solche Verhältnisse müssen, $\mathrm{n}$ a ch der interglazialen Verwitterung der Rißmoränen, also im weiteren Gebiete schon einmal geherrscht haben, bevor das Hauptwürm die Rißmoränen mit ihrer interglazialen Verwitterungsdecke und ihrer würmzeitlichen Lößdecke überwältigen konnte. Die genaue Schilderung der einschlägigen Aufschlüsse findet sich in den "Alpen im Eiszeitalter" S. 155. E. BRǗCKNER hat diese wichtigen Profile schon vor mehr als $60 \mathrm{Jah}$ ren beobachtet und gedeutet. Ich selbst konnte in früheren Jahren noch einiges davon sehen und den Befund bestätigen. Ich konnte aber auch noch neue Aufschlüsse dazu finden. Die alten sind heute weitgehend verfallen. Einer dieser neuen Aufschlüsse lag an der Ansatzstelle der Niederterrasse bei Haunsberg am Wege nach Haselreith.

Es handelt sich also bei der Erhaltung von Resten einer älteren Lößlehmdecke in den Basisschichten von Hauptwürm um ein regionales Phänomen. Dies ergibt cbenfalls 
einen Hinweis auf ein älteres, weiter zurückgelegenes Würmstadium mit lößbildenden Eigenschaften. Penck-Brückner erwähnen ein Gegenstück hierzu für das Gebiet von Lyon im Rhônegletscher.

Zusammenfassung über Ơlkofner Phase und überfahrenes Würmstadium. Die oben dargelegten Gründe führen dazu, neben einer frischen Lanzinger (Olkofner) Phase auch noch ein älteres, überfahrenes Würmstadium anzunehmen, welche beide räumlich eng beisammen, stellenweise auch übereinander liegen. Auf dem westlichen Salzachgletscherflügel liegt die Lanzinger Phase distalwärts des überfahrenen Würmstadiums unmittelbar am Ostrande des Ollerdinger Eisrandtales. Sie läßt sich von mindestens Burg bei Tengling bis Lanzing und Tittmoning hin verfolgen.

Anschließend an einen Vortrag der Verf. über das Thema dieses Aufsatzes vor dem Geologisch-mineralogischen Arbeitskreis beim "Haus der Natur" in Salzburg am 7. 10. 1954 und eine gemeinsame Besichtigung der bayrischen Aufschlüsse konnte W. DEx NEGro, Salzburg, einen wertvollen Beitrag liefern. DEL NEGRo schreibt hierzu: „Das kleine Zweigbecken des Salzachgletschers im Raum Kraiwiesen-Unzing an der Bahnlinie Salzburg-Ischl wird von drei Jungmoränenwällen umschlossen (Abb. 2). Von diesen repräsentiert aber der äußerste, wie L. WeINBERGER erkannt hat, die Kirchseeoner und Ebersberger Phase zusammen, deren Endmoränenwälle östlich des Wallersees noch getrennt verlaufen, nach Süden zu aber konvergieren und sich dann gemeinsam um die Flyschhänge des Zifanken schlingen. In Stirnberührung mit den Endmoränen eines Traungletscherarmes umziehen sie das erwähnte Zweigbecken. Der nächstinnere Wall macht einen überschliffenen Eindruck und ist daher wohl KNAUER's W I zuzuweisen. Der innerste Wall wurde schon von L. WEINBERGER als Rückzugsgebilde gedeutet, worauf auch mehrere Oser beiderseits des Unzinger Mooses hinweisen. Er wäre daher der Olkofener Phase zuzuweisen, deren Moränen hier also innerhalb der W I-Moränen lägen. Sie sind von diesen durch ein peripheres Tal getrennt. - In der nördlichen Fortsetzung entspricht aber diesen beiden Wällen ein einziger Wall, in welchem Ölkofner Phase und W I zusammenfallen. Ein Aufschluß (Abb. 3) bestätigt dies. Uber basalen verfestigten Deltaschottern, deren obere Grenzfläche infolge Abtragung unregelmäßig verläuft, folgt eine sehr grobblockige Moräne, die gegen das Hangende durch eine flach südfallende scharf ausgeprägte Diskordanz mit Konglomeratbänkchen begrenzt wird. Oberhalb dieses Bänkchens liegt eine Jungmoräne mit bedeutend weniger groben $\mathrm{Ge}$ schieben. Damit stellt dieser Aufschluß ein Gegenstück zu dem von Oberstefling im
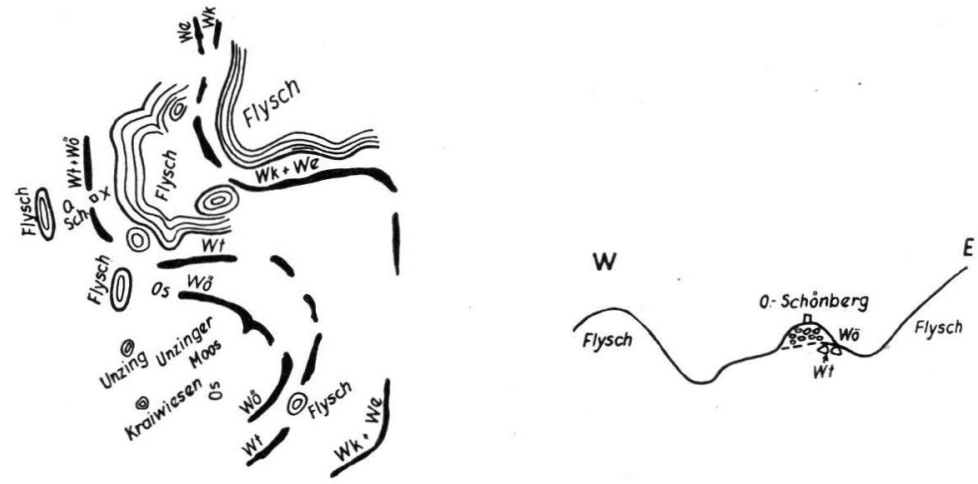

Abb. 2 (links). Endmoränenlagen im Bereich von Kraiwiesen (Ostflügel des Salzachgletschers)

$\mathrm{Wö}=$ Ớlkofener Phase; We = Ebersberger Phase; Wk = Kirchseeoner Phase; Wt = Tenglinger Stadium; O Sch = Ober-Schönberg; $X=$ Lage des Aufschlusses Abb. 3. Abb. 3 (rechts). Profil des Walles von Ober-Schönberg. Signaturen wie Abb. 2. 
bayrischen Sektor dar, der von E. EBERS als überfahrene W I-Moräne mit hangender Hauptwürm-Moräne gedeutet wurde. Bei Kraiwiesen kommt aber noch hinzu, daß in der genauen Fortsetzung der durch das Konglomeratbänkchen markierten Grenzfläche südlich außerhalb des Aufschlusses ein Geländeknick durchzieht, der den Steilhang des Walles, auf dem Ober-Schönberg steht, von einem nach unten anschließenden Flachhang trennt. Der im Aufschluß sichtbare stratigraphische Unterschied der beiden Moränen wird also außerhalb des Aufschlusses durch einen genau korrespondierenden morphologischen Gegensatz ergänzt: der ausgeprägte W'all mit seinem Steilhang gehört offensichtlich zur Ólkofner Phase, der Flachhang darunter, dem am Aufschluß die grobblockige Moräne unterhalb des Konglomeratbänkchens zugeordnet ist, zur überfahrenen W I-Moräne. Während im südlich anschließenden Gebiet des Zweigbeckens von Kraiwiesen-Unzing die Moränen von Olkofner Phase und W I nebeneinander zu liegen kamen, sind sie hier im Raum von Ober-Schönberg superponiert. “

Wenn man die langjährige Diskussion über das „überfahrene Würmstadium“ zwischen J. Knauer und C. Troll u. a. nachliest, so findet man als Hauptargument immer wieder die Frage nach "frischen Sandern" auf der Außenseite der fraglichen Moränen. Man kann J. KNAUER recht geben, daß die zu einem überfahrenen Würmstadium gehörigen Schotterkörper verstümmelt sein müssen und in dieser Form auch zu finden sind. Eine stratigraphische Verknüpfung von Moräne und Sander, die C. Troll (1936) mit Recht fordert, wird sich im Untersuchungsgebiet aber kaum nachweisen lassen. Das erst spätglazial angelegte Salzachtal Tittmoning-Burghausen ist kein entsprechendes Gegenstück zum Inntal. Durchlaufende liegende ältere Schotter unter den Hauptwürmschottern dürften auch im österreichischen Weilhartforst schwer nachzuweisen sein, weil dort ein entsprechend tiefer Taleinschnitt fehlt. Die Sander der Ölkofner Phase sind in fast allen nordalpinen Vorlandgletschern durch periphere Talzüge ersetzt und durch die zugehörigen Deltas der ältesten und obersten Stauseen. Erst an der Spitze des Hauptbeckenzuges bringt eine große Hauptsammelader die Schmelzwässer nach außen. Wie schon erwähnt, muß das der Zeitabschnitt der Ebinger Stufe gewesen sein, und es müssen Vorgänge stattgefunden haben, die eine einschneidende Bedeutung für die Gliederung der Würmeiszeit haben. Denn hinter ihnen muß eine großklimatische Anderung stehen.

\section{Die Frage der älteren Würmschotter und das Aurignac-Interstadial}

La ufenschotter, Altere Würmschotter. Ein Punkt bedarf in diesem Zusammenhang einer noch etwas eingehenderen Betrachtung: es ist das jahrzehntealte Problem des sog. Laufenschotters und der älteren Würmschotter überhaupt. Für manche Autoren sind die "liegenden" oder „unteren" Würmschotter identisch mit dem „Laufenschotter". J. KNAUER unterscheidet „untere Würmschotter" (Laufenschotter; als Vorrückungsschotter von Würm) und die Schotter der W I-Phase. Inwieweit solche Gleichsetzungen zutreffen, läßt sich bis heute noch nicht sicher beurteilen. Die nachfolgenden Darlegungen haben daher nur vorläufigen Charakter, bemühen sich aber, das schon Gesicherte hervorzuheben. Der Laufenschotter im engeren Sinn und oberen Teil schält sich danach jetzt mehr und mehr als Vorrüickungsschotter von Hauptwürm heraus.

Penck-BrüCKner haben nach dem Laufenschotter ihre „Laufenschwankung“ benannt und sie in den Höhepunkt der Würmvereisung gestellt. A. PENCK hat sie 1922 wieder aufgegeben. Hierzu haben O. Ampferer's (1907-1909) Untersuchungen im alpinen Inntal beigetragen, der dort nur interglaziale, keine interstadialen Bildungen erkennen konnte. In den großen Tälern der nördlichen Alpenfront findet sich vielfach eine einheitliche Formation, die aus Schottern, Sanden und Tonen besteht und zwischen zwei Moränen eingelagert ist. Der klassische Laufenschotter von Laufen-Oberndorf im Salz- 
achtal wird 1922 von A. PENCK garnicht mehr erwähnt und ebensowenig die zahlreichen früher gewonnenen Einzelergebnisse rückgängig gemacht. A. PENCK und E. BRüCKNER hatten sie an zahlreichen anderen Stellen gefördert und ein interstadiales Alter des Laufenschotters daraus abgeleitet. F. MachatscheK hält 1935/36 die Frage des Laufenschotters für noch ungeklärt.

Heute frägt man sich: worauf anders weisen die weithin überfahrenen FrühwürmAblagerungen eigentlich hin, wenn nicht auf einen beträchtlichen Gletscherrückzug, ein Interstadium, eine sog. Schwankung? Der Laufenschotter kann sich nur auf eisfreiem Gebiet abgelagert haben. Im Salzachgebiet bei Laufen findet sich der Prototyp des Laufenschotters, eine Bildung, welche sich meist am Rand der Tiefenzüge, aber auch in großen Schotterfetzen seitlich der Becken verbreitet. Sie liegt zwischen zwei Moränen, von welchen die obere einer regional verbreiteten Grundmoränendecke angehört, die alle älteren Würmbildungen proximalwärts der Hauptwürm-Endmoränen verhüllt und auch die Drumlins überzieht oder ganz aufbaut. Sie ist konkordant gelagert und häufig - nicht immer - mit dem Laufenschotter verknüpft. Sie gehört dem Hauptwürm an.

Nach Art seiner bankweisen Verfestigung, die von L. SIMON (1935) mit Recht als eine "Grundwasserverfestigung bei fortschreitender Talbildung" angesehen wird, also eine "Verfestigung von unten“ an Stelle derjenigen „von oben“, wie sie die diagenetische Verfestigung mittel- und altpleistozäner Schotter darstellt, ähnelt er den anderen Frühwürmablagerungen. Auch die geographische Lage der Schotterkomplexe, die meist auffallend nahe an den Alpenrand herangehen, ist für sie bezeichnend. In dieser Lage ist er in der Mehrzahl der voralpinen Gletscherzungen Deutschlands und der Schweiz erhalten geblieben.

Über die immer wieder auftretende „liegende Moräne“ (z. B. bei Laufen, im Inngebiet, in der Schussenzunge des Rheingletschers) ist es natürlich schwer, bestimmte Aussagen $\mathrm{zu}$ machen. Bei Laufen-Oberndorf ähnelt sie sehr viel mehr einer Würm- als einer Riß-Grundmoräne. Noch deutlicher und sicherer wird das im Rheingletschergebiet. Es sind dort im Laura- und im Flattbachtal bei Ravensburg und bei Markdorf am Göhrenberg beträchtliche Schotterreste erhalten, die zwischen zwei Moränen liegen, von denen auch die liegende erhebliche Mächtigkeiten erlangen kann. M. SснмIDT (1931) zufolge ist auch die liegende Moräne unzweifelhaft und aus mehreren Gründen zu Würm zu rechnen. Schon 1911 machte M. SCHMIDT darauf aufmerksam, daß im Argental mächtige Altwürm-Moränen im Liegenden von Fluvioglazialschottern mit Grundmoränendecke $\mathrm{zu}$ finden sind. Ein rißeiszeitliches Alter komme keinesfalls für sie in Betracht, da alle Rißbildungen $200 \mathrm{~m}$ höher lägen.

Auch nach den PENCK'schen Darlegungen von 1922 kann der Laufenschotter - bei seiner gelegentlichen Verknüpfung mit den oberen Moränen und den Anzeichen eines kalten Klimas in den oberen Lagen - nicht ganz als interglaziale Bildung im Sinne PenCK-BrüCKNER's angesehen werden. Im klassischen Laufenschotter des Salzachgletschergebietes fehlen ihm auch eine lakustre Phase und Schieferkohlen bislang. Was ihn noch besonders auszeichnet und oben als kalten Schotter charakterisiert ist, daß in ihm (wie bei Markdorf, Rheingletscher) bei Spöckmühle am Waginger See Grundmoränenbänke auftreten. In der Hauptachse des Gletschers, bei Laufen-Oberndorf, besitzt er allerdings betont fluviatilen Charakter mit Dachziegellagerung, was aber nur eine fazielle Verschiedenheit darstellen dürfte. Außerdem stecken in diesem Schotter unter der Grundmoränendecke gelegentlich auch Eiskeile. Der beste bisher bekannt gewordene ${ }^{3}$ ) ist vom epigenetischen Typ, mit Sand, Feinkies und Tonlagen verfüllt und $2 \mathrm{~m}$ lang und ebenso breit. Es spricht aber noch eine zweite sehr bedeutsame Tatsache für den

3) Bei Spöckmühle. 
„kalten“ Schotter. Er und auch die übrigen älteren Würmschotter sind Mammutschotter "par excellence“. Bei Laufen-Oberndorf fanden sich in den Steinbrüchen vier Molaren (jetzt in Salzburg "Haus der Natur") in $36 \mathrm{~m}$ Höhe über der Salzach (nach FugGer 1899). A. Penck erwähnt von Gabersee, Lauterbach und Landl, alle bei Rosenheim im Inngletschergebiet, Elephas primigenius in den entsprechenden Schottern. Nach P. BECK (1938) fand sich im "Münsingen"- sprich Laufenschotter des Aaregebietes ein Mammutmolar. In den entsprechenden Eschenbachschottern des Reußgletschers kommen nach A. BöGLI (freundliche mündliche Mitteilung) ebenfalls Mammutmolare vor. Praktisch sind, bei näherer Nachprüfung der Provenienz, fast alle Funde von Mammutstoßzähnen und Mammutmolaren, die innerhalb des ehemals vereisten nôrdlichen Alpenrandgebietes gemacht wurden, an den Laufenschotter oder sonstige, von ihm noch nicht abtrennbare ältere Würmschotter geknüpft. Diese Häufung des Mammuts in den Schottern von Frühwürm und insbesondere im Laufenschotter ist auffällig. Nach Soengel (1940) ist die Kälteanpassung des Mammuts eine vollkommene gewesen und es trug einen ausgesprochen stenothermen Charakter. Das Mammut verschwand nach SoERGEI in interglazialen Zeiten und zog sich in Interstadialen in die Nähe des Eisrandes zurück. Da sich Mammutzähne in Frühwürmschottern und besonders im Laufenschotter in der Nachbarschaft des Gebirgsrandes immer wieder finden, würde das bedeuten, daß die Alpen zur Zeit der Bildung des Laufenschotters schon wieder oder noch stark vergletschert waren.

A. G. Stehlin und E. Schertz, die den Münsingen-Zahn genau untersuchten und ihn mit von Artefakten begleiteten Faunen verglichen, kamen zu dem Schluß, daß er ins Mittelaurignacien zu stellen sei. Die Begründung hierfür liegt nach A. Schertz (1938) in den Ergebnissen eines Vergleiches mit den Molaren aus dem „Mammutlager“ der Vogelherdhöhle im Lonetal/Alb. Die stratifizierten Stücke dieser Höhle lassen eine Entwicklung ablesen in dem Sinne, daß nach oben die Zahl der Lamellen im Zahne zunimmt ebenso wie die Zahnhöhe. Die Lamellen werden auch in der Längserstreckung des Zahnes kürzer. Dieselbe Elephas primigenius-Form tritt bei Hangenbieten-Achenheim am Ausgange des Breuschtales W Straßburg zusammen mit Aurignacien zwischen den beiden jüngsten Lössen auf. An Hand dieser Befunde erscheint es doch als zweckmäßig, nunmehr alle in „älteren Würmschottern" aufgefundenen Mammutmolare mit dem Münsingen-Exemplar zu vergleichen.

Laufenschwankung oder Aurignac-Interstadial. Wenn das von österreichischen (H. GAMs, Inqua 1953) und schweizerischen (W. LüDI 1953) Paläobotanikern ebenso wie von P. BECK aufgestellte Postulat eines Aurignac-Interstadials auch im Alpenraum zutrifft, so vermag dies zur Wiederaufnahme der Laufenschwankung Penck-Brückner's zu führen. P. Beck hat schon im Jahre 1933 die Spiezer Schwankung als Laufenschwankung wiedererkannt. Als eine Schwierigkeit bleibt vorläufig bestehen, daß seine Spiezer Schwankung zwischen den ersten, am weitesten ins Vorland vorgedrungenen Vorstoß des Aaregletschers, den Gurten-Vorstoß, und einen zweiten, weiter zurückgebliebenen Vorstoß, das Berner Stadium, eingeordnet wird. Diese Schwierigkeit besteht dann, wenn Gurten und Berner Vorstoß mit der Kirchseeoner und Ebersberger Phase gleichzusetzen wären. A. BöGLI kennt im Seental des Reußgletschers eine stadiale Schwankung, die er nicht nach, sondern vor den Maximalstand der WürmVereisung einordnen kann. J. KNAUER $(1938,1954)$ findet ein überfahrenes Würm IStadium auch für die Schweiz am Linth- und Reußgletscher.

In der letzterschienenen Veröffentlichung von W. LüDI (1953) werden mehrere der Schweizer Schieferkohlenlagerstätten auf Grund pollenanalytischer Verhältnisse zu einem bedeutenden frühen Würm-Interstadial gerechnet, das einen weiten Rückzug, kalt-feuchtes Klima und sogar Wiederbewaldung mit sich brachte. Die pollenanalytischen Untersuchungen von H. REICH (1953) im bayrischen Alpenvorlande, welche eine Deutung der Schieferkohlen von Großweil-Ohlstadt (Isargletscher) und Pfefferbichl 


\section{Der Ablauf der Würmvereisung im Salzach- und Innvorlandgletschergebiet}

Versuch einer Gliederung und Parallelisierung

\begin{tabular}{|c|c|c|c|c|}
\hline & Salzachgletscher & Inngletscher & $\begin{array}{l}\text { Schotterstufen } \\
\text { im Inngebiet }\end{array}$ & $\begin{array}{l}\text { Nordische } \\
\text { Vereisung }\end{array}$ \\
\hline $\begin{array}{l}\text { Früh- } \\
\text { Würm }\end{array}$ & $\begin{array}{l}\text { Tenglinger Stad. } \\
\text { Rückzug ins Gebirge } \\
\text { Aurignac-Interstad. }\end{array}$ & $\begin{array}{l}\text { Ubberfahr. W I-Mor. } \\
\text { Rückzug ins Gebirge } \\
\text { Aurignac-Interstad. }\end{array}$ & . & $\begin{array}{l}\text { Stettiner } \\
\text { überf. Moräne } \\
\text { Aurignac-Interstad. }\end{array}$ \\
\hline $\begin{array}{l}\text { Haupt- } \\
\text { Würm }\end{array}$ & $\begin{array}{l}\text { Unterweißenkirch. } \\
\text { Außenphase } \\
\text { Nunreuther } \\
\text { Hauptphase } \\
\text { Radegunder } \\
\text { Hauptphase }\end{array}$ & $\begin{array}{l}\text { Ayinger } \\
\text { Außenphase } \\
\text { Kirchseeoner } \\
\text { Hauptphase } \\
\text { Ebersberger } \\
\text { Hauptphase }\end{array}$ & $\begin{array}{l}\text { Neustadler } \\
\text { Aicher } \\
\text { Trauntaler } \\
\text { Rauschinger }\end{array}$ & $\begin{array}{l}\text { Brandenburger } \\
\text { Stadium } \\
\text { Frankfurter } \\
\text { Stadium }\end{array}$ \\
\hline $\begin{array}{l}\text { Spät- } \\
\text { Würm }\end{array}$ & $\begin{array}{l}\text { Lanzinger Phase } \\
\text { mit Ollerdinger } \\
\text { Eisrandtal } \\
\text { Tittmoninger Eis- } \\
\text { stausee (465-475?) } \\
\text { Schmelzwasserseen } \\
\text { I (440 m) } \\
\text { II (410-420 m) } \\
\text { v. Salzburg-Titt- } \\
\text { moning } \\
\text { Rückzuggmoränen } \\
\text { von Pfarrhof b. } \\
\text { Teisendorf }\end{array}$ & $\begin{array}{l}\text { Ólkofner Phase } \\
\text { mit Leizach-Gars- } \\
\text { Talzug. } \\
\text { Edlinger Eisstausee } \\
\text { (obere Stufe) } \\
\text { Rosenheimer See } \\
\\
\text { Rückzugsmoränen } \\
\text { von Stephanskirchen }\end{array}$ & $\begin{array}{l}\text { Ebinger } \\
\text { Wörther } \\
\text { Pürtner }\end{array}$ & $\begin{array}{l}\text { Baltischer Eissee } \\
\text { usw. }\end{array}$ \\
\hline $\begin{array}{l}\text { End- } \\
\text { Würm }\end{array}$ & & & $\begin{array}{l}\text { Gwenger } \\
\text { Niederndorfer }\end{array}$ & Salpausselkä I-III \\
\hline
\end{tabular}

(Gliederungen nach C. Troll 1924, P. Woldstedt 1950, J. Knauer 1935, W. Koenne 1916). (Lechgletscher) als interglazial ermöglichen, haben allerdings keine Hinweise auf dies Interstadial gegeben. Im hangenden Schotter fand sich ein Mammutzahn.

Einordnung des Aurignac-Intarstadials in globale Zusammenhänge. Wie sich dieses Interstadial in die globalen Zusammenhänge einordnen würde, zeigen die jüngsten Veröffentlichungen von P. WoLdstedt (1950) für die nordeuropäische und von R. F. FLINT (1953) für die nordamerikanische Vereisung. Auch in Norddeutschland finden sich neuerdings Anzeichen für eine überfahrene Vorrückungsphase. Es handelt sich dabei um die Stettiner Stauchmoränen, die eine Grundmoränendecke tragen. Dem anschließenden Interstadial (Aurignac-Interstadial?) gehört wahrscheinlich die Rixdorfer Fauna (bei Berlin), wieder eine Elephas-primigenius-Fauna, an. Das Aurignac-Interstadial entspricht der Hauptverlehmungszone im „Jüngeren Löß“. Es liegt zeitlich zwischen dem überfahrenen Vorrückungsstadium und dem Brandenburger Stadium. Dem Aurignac-Interstadial entspricht in Nordamerika das Brady-Intervall, das durch Bodenbildungen und überfahrene Wälder gekennzeichnet ist. M. PFanNENSTIEL (1951) rechnet im östlichen Mittelmeergebiet dem „Würm I-Stadium“ die posttyrrhennische Regression zu, mit einer Absenkung des Meeresspiegels von etwa $100 \mathrm{~m}$.

Ungeklärte Fragen. Außer der noch nicht übereinstimmenden Einordnung der Spiezer Schwankung P. BECK's stellt sich einer Wiederaufnahme der Laufenschwankung im nördlichen Alpenvorland, wie schon erwähnt, die Auffassung O. AmpFerer's 
(1907-1909) entgegen - und die A. Penck's seit 1922 - daß in dem großen Komplex der "Mittelterrasse“ des inneralpinen Inntales nur interglaziale und nicht interstadiale Bildungen $\mathrm{zu}$ sehen sind. A. Penck übertrug diese Auffassung auch auf die übrigen Alpentäler, und die österreichischen Quartärforscher (SEEFELDNER, PIPPAN u. a., nach freundlicher mündlicher Mitteilung) halten mit guten Gründen daran fest, auch für das inneralpine Salzachtal. Besonders die große Mächtigkeit der einschlägigen Bildungen $(300 \mathrm{~m})$ spricht dort mehr für ein Interglazial als für ein Interstadial, ohne entscheidend $\mathrm{zu}$ sein. Tektonische Vorgänge haben im Salzachtal mitgewirkt. Wenn z. Zt. der Laufenschotterablagerung die Alpen bis an den Rand vereist waren, wofür das gehäufte Auftreten des Mammut spricht, so ist das Fehlen des Laufenschotters im inneralpinen Salzachtal wohl erklärbar. Nach einer freundlichen brieflichen Mitteilung von P. BECK, 1954, hat die Bildung der Spiezer Interstadialschotter (Laufenschotter) einen Eisschwund von $600-650 \mathrm{~m}$ zur Voraussetzung. Nimmt man einen ähnlichen Wert für das Salzachgebiet an, so müßte die Eisoberfläche in der Gegend von Salzburg nur mehr in $400-450 \mathrm{~m}$ gelegen haben (Stadt Salzburg $425 \mathrm{~m}$ ).

\section{Schriftenverzeichnis}

AmpFerer, O.: Glazialgeologische Beobachtungen im unteren Inntal. - Zs. f. Gletscherkunde 2, 1907/08. - - Uber die Entstehung der Inntalterrassen. - Ebendort 3, 1908/09.

BECK, P.: Úber den eiszeitlichen Aaregletscher. - Mitt. naturw. Ges. Thun 1932. - - Über das schweizerische und das europäische Pliozän und Pleistozän. - Ecl. geol. Helv. 26, 1933. - - Bericht über die außerordentliche Frühjahrsversammlung der Schweiz. Geol. Ges. in Thun 1938. - Ebendort 31, 1938.

Brǘckner, E.: Die Vergletscherung des Salzachgebietes. - Geogr. Abh. 1, Wien 1886.

BüDEL, J.: Die Klimaphasen der Würmeiszeit. - Die Naturwissenschaften 37, 1950.

Ebers, E.: Über erloschene Seen im Salzachgletschergebiet. - Mitt. Geogr. Ges. München 25, 1932.

FLINT, R. F.: Recent advances in North American Pleistocene stratigraphy. - Eiszeitalter und Gegenwart 3, 1953.

FugGer, E.: Das Salzburger Vorland. - Jb. k. k. geol. R. A. 49, 1899.

GRIPP, K.: Müssen gewisse jungeiszeitliche Endmoränenzüge im nördlichen Alpenvorland und in Norddeutschland als vom Eise überfahren angesehen werden? - Mitt. geogr. Ges. Lübeck 40, 1940.

Knauer, J.: Die Ablagerungen der älteren Würm-Eiszeit (Vorrückungsphase) im süddeutschen und norddeutschen Vereisungsgebiet. - Abh. geol. Landesuntersuchung am bayerischen Oberbergamt 21, München 1935. - - Widerlegung der Einwendungen K. TroLL's gegen die Vorrückungsphase der Würm-Eiszeit. - Mitt. geogr. Ges. München 30, 1937. - Über das Alter der Moränen der Zürichphase im Linthgletschergebiet. - Abh. geol. Landesuntersuchung am bayerischen Oberbergamt 33, München 1938. - - Zur Theorie der überfahrenen Würm-Moränen. - Mitt. d. Reichsstelle f. Bodenforschung 34, 1941. - - Über die zeitliche Einordnung der Moränen „Zürich-Phase“ im Reußgletschergebiet. - Geogr. Helv. 2, 1954.

Koenne, W.: Bl. Ampfing Nr. 675. - Erläuterungen zur Geol Karte d. Königreichs Bayern $1: 25$ 000. München 1916.

LüdI, W.: Die Pflanzenwelt des Eiszeitalters im nördlichen Vorland der Schweizer Alpen. Veröff. geobot. Inst. Rübel in Zürich 2\%, 1953.

MachatscheK, F.: Die Literatur zur alpinen Eiszeitforschung. 2, 1935/36.

Micheler, A.: Verwitterungshorizont der Würm I-Phase bei Ob im Lechgebiet. - Naturforschende Gesellschaft Augsburg 1948.

A. Penck - E. BrüCKNer: Die Alpen im Eiszeitalter. Leipzig 1909.

Pfannenstiel, M.: Quartäre Spiegelschwankungen des Mittelmeeres und des Schwarzen Meeres. - Vierteljahresschrift naturf. Ges. Zürich 96, 1951.

Rathjens, C.: Über die Zweiteilung der Würmeiszeit im nördlichen Alpenvorlande. - Pet. Mitt. 1951,

Reich, H.: Die Vegetationsentwicklung der Interglaziale von Großweil-Ohlstadt und Pfefferbichl im Bayerischen Alpenvorland. - Flora 140, 1953.

Schaefer, J., Graul, H., Brunnacker, K.: Zur Gliederung der Würmeiszeit im Illergebiet. Geol. Bav. 18, 1953.

ScherTz, E.: Bericht über die außerordentliche Frühjahrsversammlung der Schweiz. Geol. Ges. in Thun, 1938, S. 189. 
SснміDт, M.: Rückzugsstadien der Würmvergletscherung im Argengebiet. - Schriften des Vereines f. Geschichte d. Bodensees 40, Lindau 1911. - - Erläut. geol. Spezialkarte von Württemberg, Bl. Ravensburg, Nr. 175, Stuttgart 1931.

SeEfeldner, E.: Entstehung und Alter der Salzburger Ebene, - Mitt. Ges. f. Salzburger Landeskunde 94, 1954.

Simon, L.: Kleine Beobachtungen am Laufenschotter des Salzachgletschergebietes. Ein Beitrag zur Nagelfluhbildung. - Abh. geol. Landesuntersuchung bayr. Oberbergamt 18, 1935.

SoErgel, W.: Der Klimacharakter des Mammut. - Paläont. Zs. 22, 1940.

Troll, C.: Der diluviale Inn-Chiemseegletscher. - Stuttgart 1924. - - Die sog. Vorrückungsphase der Würm-Eiszeit und der Eiszerfall bei ihrem Rückgang. - Mitt. geogr. Ges. Ges. München 29, 1936.

Woldstedt, P.: Norddeutschland und angrenzende Gebiete im Eiszeitalter. - Stuttgart 1950.

Einschlägige Kartenblätter des Topogr. Atlas von Bayern $1: 25000$ Nr. 746, 747 (TachingerWaginger See) und anschließende B!ätter (Nr. 723, 724, 745).

Manuskr. eingeg. 10. 1. 1955.

Anschrift der Verfasserin: Dr. Edith Ebers, Haunshofen bei Stat. Diemendorf, Oberbayern. 